Pacific Journal of Mathematic 


\title{
BANACH SPACES WITH A RESTRICTED HAHN-BANACH EXTENSION PROPERTY
}

\author{
Charles W. Neville
}

\begin{abstract}
We shall study the class of real Banach spaces $B$ with the following restricted Hahn-Banach extension property: For each Banach space $C$ with a dense set of cardinality $\leqq$ some fixed cardinal $\mathfrak{N}$, and for each subspace $A$ of $C$ and bounded linear map $T_{0}: A \rightarrow B$, there exists an extension $T: C \rightarrow B$ such that $\|T\|=\left\|T_{0}\right\|$. Suprisingly, there exist Banach spaces in this class which are not isometrically isomorphic to $C(X)$ for a compact Hausdorff $X$ !
\end{abstract}

The combined results of Goodner, Hasumi, Kelley and Nachbin show that those Banach spaces with the Hahn-Banach extension property, that is, those Banach spaces which are injective in the category $\mathscr{B}_{1}$ of Banach spaces and linear maps of norm $\leqq 1$, are precisely the Banach spaces of the form $C(X)$, where $X$ is compact Hansdorff and extremally disconnected [5], [6], [7], [11]. In this paper, we wish to study those Banach spaces which enjoy a restricted Hahn-Banach extension property, where the existence of an extension is only required for spaces which are relatively small.

To be more precise, let $\mathfrak{N}$ be an infinite cardinal. We shall say that a Banach space $C$ is $\Re$-separable if $C$ has a dense subset of cardinality $\Re$. As usual, the word "separable" standing alone means $\mathfrak{N}_{0}$ separable. We shall call a Banach space $B$ R-injective if $B$ has the following restricted Hahn-Banach extension property: Let $C$ be an $\mathfrak{R}$-separable Banach space, let $A$ be a subspace of $C$, let $i: A \subset C$ be the inclusion map, and let $T_{0}: A \rightarrow B$ be a bounded linear map. Then there exists a bounded linear map $T$ with $\|T\|=\left\|T_{0}\right\|$, making the following diagram commute:

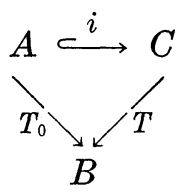

We shall study the $\mathfrak{R}$-injective Banach spaces in this paper. We shall only consider real Banach spaces here. We shall characterize

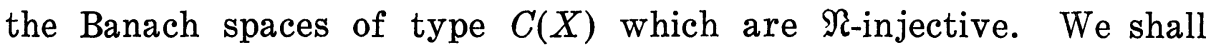
also show that there are a good many other $\mathfrak{R}$-injective Banach spaces! Finally, we shall show that if an $\mathfrak{R}$-injective Banach space also happens to be $\mathfrak{N}$-separable, then it is in fact injective in the full category $\mathscr{B}_{1}$. This contrasts rather sharply with the situation 
in the category $\mathscr{B}$ of Banach spaces and bounded linear maps. Sobczyk showed that $c_{0}$ satisfies diagram 1 , with $c_{0}=B$ and $\mathfrak{R}=\mathfrak{N}_{0}$, except that the extension $T$ may have a larger norm than $T_{0}$. On the other hand, Phillips showed that there is no continuous linear projection of $m$ onto $c_{0}$, so $c_{0}$ is not injective in the full category $\mathscr{B}$ (cf. [3, p. 25], [13]).

\section{$\Re$-Injectives of Type $C(X)$.}

First, let us prove a theorem which will enable us to characterize the $\mathfrak{R}$-injective spaces of type $C(X)$. To motivate this theorem, the reader should recall the Stone-Nakano theorem, which says, among other things, that a compact Hausdorff space $X$ is extremally disconnected if and only if $C(X)$ is a boundedly complete vector lattice under the usual ordering [12]. Thus, the Goodner-Hasumi-KelleyNachbin theorem may be rephrased to assert that the injectives in $\mathscr{B}_{1}$ are exactly the $C(X)$ 's which are also boundedly complete vector lattices. It is thus not surprising that a property similar to lattice

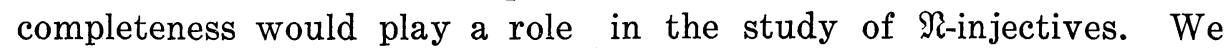
shall say that an ordered normed linear space $B$ satisfies condition $a_{\Re}$ if the following is true:

$\left(a_{\mathfrak{n}}\right)$ For each $\mathfrak{R}$-separable subspace $V$ of $B$, the following is true: Given a subset $\mathscr{F}$ of $V$ which is bounded above in norm by $m$ and of cardinality $\leqq \mathfrak{N}$, there exists at least one $b \in B$ such that $\|b\| \leqq m, f \leqq b$ for all $f \in \mathscr{F}$, and if $v \in V$ and $f \leqq v$ for all $f \in \mathscr{F}$, then $b \leqq v$.

THEOREM 1. Let $X$ be a compact Hausdorff space and let $B$ be a closed subspace of $C(X)$. Then $B$ is $\mathfrak{N}$-injective if $B$ satisfies property $a_{26}$.

Conversely, suppose $B$ is $\mathfrak{R}$-injective, and suppose $B$ contains a subset $Q$ with the following properties. $Q$ consists of nonnegative functions none of which are identically $0, Q$ contains a dense set of carinality $\leqq \mathfrak{N}$, and the set of points at which the function in $Q$ attain their suprema is dense in $X$. Then $B$ satisfies condition $a_{\mathfrak{r}}$.

Proof. First suppose that $B$ satisfies condition $a_{\text {n }}$. The proof that $B$ is $\Re$-injective follows Goodner's idea of replacing real valued sublinear functionals with $\mathrm{C}(X)$ valued sublinear functionals in Banach's original proof of the Hahn-Banach theorem [3, pp. 135137], [5]. Let $A$ be a subspace of $C$, let $C$ be $\Re$-separable, and let $T_{0}: A \rightarrow B$ be a bounded linear map. Let $p: C \rightarrow B$ be defined as follows: Let $\mathscr{K}$ be a dense subset of cardinality $\leqq \mathfrak{N}$ of the unit ball of $A$. We know that the set $T_{0}(\mathscr{K})$ has cardinality $\leqq \mathfrak{R}$ and 
is bounded above in norm by $\left\|T_{0}\right\|$. Clearly $T_{0}(A)$ is $\mathfrak{R}$-separable. By condition $a_{\mathfrak{n}}$, there exists $u \in B$ which bounds $T_{0}(\mathscr{K}) \cup\{0\}$ from above, and which satisfies $\|u\| \leqq\left\|T_{0}\right\|$. Because $\mathscr{K}$ is dense in the unit ball of $A$, we have $T_{0}(c) \leqq\|c\| u$ for all $c \in C$. Let $p(c)=\|c\| u$. Then $p$ is a sublinear map, and $T_{0}$ is dominated by $p$. Furthermore, if $S$ is any linear map from a subspace of $C$ to $B$ which is dominated by $p$, then $S$ is continuous. In fact, $S(\mathrm{c}) \leqq\|c\| u$ and $-S(c)=$ $S(-c) \leqq\|c\| u$, so $\|S\| \leqq\left\|T_{0}\right\|$.

Now suppose $A^{\prime}$ is a proper subspace of $C$ containing $A$, and suppose $T^{\prime}$ is an extension of $T_{0}$ to $A^{\prime}$ which is dominated by $p$. Let $\mathscr{K}$ be a dense subset of the unit ball of $A^{\prime}$ of cardinality $\leqq \mathfrak{R}$. Let $z \in C \sim A^{\prime}$. As in Banach's proof of the Hahn-Banach theorem, we obtain for each $(x, y) \in A^{\prime} \times A^{\prime},-p(-y-z)-T^{\prime}(y) \leqq p(x+z)-$ $T^{\prime}(x)$. Let $V=$ the linear hull of $\left\{-p(-y-z)-T^{\prime}(y): y \in A^{\prime}\right\} \cup$ $\left\{p(x+z)-T^{\prime}(x): x \in A^{\prime}\right\}$. The continuity of $p$ and $T^{\prime}$ together with the $\mathfrak{N}$-separability of $A^{\prime}$ implies that $V$ is also $\mathfrak{N}$-separable. We would like to apply condition $a_{\Re}$ to a set $\mathscr{F}=\{-p(-y-z)-$ $T^{\prime}(y): y \in$ some dense set in $\left.A^{\prime}\right\}$ to obtain the existence of $c \in B$, such that $-p(-y-z)-T^{\prime}(y) \leqq c \leqq p(x+z)-T^{\prime}(x)$ for all $(x, y) \in A^{\prime} \times A^{\prime}$. But such a set $\mathscr{F}$ would not be bounded in norm, so we shall consider a sequence of $\operatorname{sets} \mathscr{F}_{n}=\left\{-p(-y-z)-T^{\prime}(y): y \in n \mathscr{K}\right\}, n=$ $1,2, \cdots$. Since $\mathscr{K}$ is a subset of the unit ball of $A^{\prime}$, each set $\mathscr{F}_{n}$ is bounded in norm. By condition $a_{\Re}$ applied to $\mathscr{F}_{n}$ and $V$, there exists $c_{n} \in B$ such that $w \leqq c_{n} \leqq p(x+z)-T^{\prime}(x)$ for each $w \in \mathscr{F}_{n}$ and $x \in A^{\prime}$. Since $\mathscr{K}$ is dense in $U$, the unit ball of $A^{\prime}$, we have $-p(-y-z)-T^{\prime}(y) \leqq c_{n} \leqq p(x+z)-T^{\prime}(x)$ for each $x \in A^{\prime}$ and $y \in n U$. Let $W=$ the linear hull of $V$ and $\left\{c_{n}: n=1,2, \cdots\right\}$. Pick $x_{0} \in A^{\prime}$ and $y_{0} \in U$. Since $U \leqq n U,-p\left(-y_{0}-z\right)-T^{\prime}\left(y_{0}\right) \leqq c_{n} \leqq p\left(x_{0}+z\right)-T^{\prime}\left(x_{0}\right)$ for $n=1,2, \cdots$, so the set $\mathscr{C}=\left\{c_{n}: n=1,2, \cdots\right\}$ is bounded in norm. Clearly $\mathscr{C}$ is $\mathfrak{N}$-separable, so by condition $a_{\mathfrak{R}}$ applied to $\mathscr{C}$ and $W$, there exists $c \in B$ such that $c_{n} \leqq c \leqq p(x+z)-T^{\prime}(x)$ for each $n$ and each $x \in A^{\prime}$. Hence $-p(-y-z)-T^{\prime}(y) \leqq c \leqq p(x+z)-T^{\prime}(x)$ for all $(x, y) \in A^{\prime} \times A^{\prime}$. The rest of the proof now follows from Zorn's lemma or transfinite induction exactly as in Banach's original proof (cf. [3, p. 10]).

Conversely, suppose that $B$ is $\mathfrak{R}$-injective, and that $Q \subseteq B$ contains a dense subset of cardinality $\leqq \Re$ and consists of nonnegative elements. Let $Y \subseteq X$ be the set of points at which the elements of $Q$ attain their suprema, and suppose $Y$ is dense in $X$. We wish to show that $B$ satisfies condition $a_{\mathfrak{r}}$. Let $V$ be an $\mathfrak{N}$-separable subspace of $B$, and let $\mathscr{F}$ be a subset of $V$ of cardinality $\leqq \Re$ which is bounded above by $m$ in norm. Let $f \in l^{\infty}(X)$ be the pointwise sup- 
remum of the set $\mathscr{F}$. Then $\|f\|_{\infty} \leqq m$. Let $A$ be the closed linear hull of $V \cup Q$, let $C$ be the linear hull of $A$ and $\{f\}$ in $l^{\infty}(X)$, and consider the commutative diagram

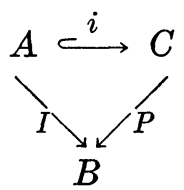

Here, $I$ is the inclusion map and $P$ is the map guaranteed by $\Re$ injectivity. We assert that $b=P(f)$ is the element whose existence is required by condition $a_{\Re}$.

Clearly it suffices to show that the map $P$ is positive. For then, if $v \in \mathscr{F}$, we have $v \leqq f$, which implies that $v=P(v) \leqq P(f)$. Also, if $v \in V$ and $v$ is an upper bound for $\mathscr{F}$, then $f \leqq v$, which implies $P(f) \leqq P(v)=v$. Finally $\|P(f)\| \leqq\|f\|_{\infty} \leqq m$. But it is easy to show that $P$ is positive. Let $c \in C$ be $\geqq 0$. Let $y \in Y$. There exists at least one $q \in Q$ such that $q \geqq 0$, and $q(y)=\|q\|>0$. Let $\lambda>0$ be such that $\|\lambda q\|=\|c\|$. Then $\|c\| \geqq\|\lambda q-c\| \geqq\|P(\lambda q-c)\|=$ $\|\lambda q-P(c)\| \geqq \lambda q(y)-P(c)(y)=\|c\|-P(c)(y)$. Thus $P(c) \geqq 0$ on $Y$. Since $P(c) \in C(X)$ and $Y$ is dense in $X$, we have $P(c) \geqq 0$ on $X$. This concludes the proof of Theorem 1 .

If we pick $B=C(X)$ and $Q=\{1\}$ in Theorem 1 , we immediately have

Corollary 1. $C(X)$ is $\mathfrak{N}$-injective if and only if $C(X)$ satisfies condition $a_{\mathfrak{n}}$.

Clearly, $C(X)$ satisfies condition $a_{\mathfrak{n}}$ for every cardinal $\mathfrak{N}$ if and only if $C(X)$ is a boundedly complete vector lattice. Thus we have another proof of the following result of Goodner and Nachbin [5], [11]:

CoRollary 2. $C(X)$ is injective in the category $\mathscr{B}_{1}$ if and only if $C(X)$ is a boundedly complete vector lattice. Equivalently, using the Stone-Nakano theorem, $C(X)$ is injective in the category $\mathscr{B}_{1}$ if and only if the compact Hausdorff space $X$ is extremally disconnected.

Of course, if $C(X)$ is a boundedly $N$-complete vector lattice, that is, if every set in $C(X)$ of cardinality $\leqq \mathfrak{N}$ which is bounded above has a least upper bound, then $C(X)$ clearly satisfies condition $a_{\mathfrak{r}}$. From the Stone-Nakano theorem again, we know that $C(X)$ is boundedly $\mathfrak{R}$-complete if and only if $X$ is both totally disconnected and 
$\mathfrak{R}$-disconnected. Here, $\mathfrak{R}$-disconnected means the closure of a union of at most $\mathfrak{R}$ clopen sets is again clopen, and the if part is true because we assume $X$ is compact Hausdorff [11], [12]. Thus we have

CoRollary 3. $C(X)$ is $\mathfrak{R}$-injective if it is a boundedly $\mathfrak{R}$-complete vector lattice. Equivalently, $C(X)$ is $\mathfrak{R}$-injective if the compact Hausdorff space $X$ is both totally disconnected and $\mathfrak{R}$-disconnected.

We do not know whether there exist spaces $C(X)$ which satisfy condition $a_{\mathfrak{n}}$, but which are not boundedly $\mathfrak{R}$-complete vector lattices. We conjecture that there are such spaces.

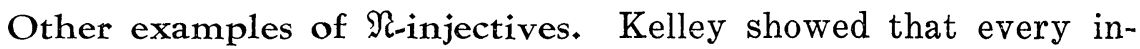
jective in the category $\mathscr{B}_{1}$ is of type $C(X)$ [7]. This is not true for $\mathfrak{N}$-injectives! We with to give a general example of a proper subspace of $C(X)$ which is $N$-injective, but which is not of type $C(Z)$ for any compact Hausdorff $Z$. To do this, we shall need an example of a compact Hausdorff space $X$ with special properties. We shall call a point $p$ of a topological space $Y$ an $\mathfrak{N}-P$ point if the intersection of $\mathfrak{N}$ neighborhoods of $p$ is again a neighborhood of $p$. The standard name for an $\mathfrak{R}_{0}-P$ point is just $P$-point (cf. [4, Chapter 4]).

First, let us manufacture an example of a compact Hausdorff space $X$ which contains a nonisolated $\mathfrak{R}-P$ point $x_{0}$, and which is totally disconnected and $\mathfrak{R}$-disconnected, but not extremally disconnected. For $\mathfrak{N}=\mathfrak{N}_{0}$, such examples are fairly ubiquitous in point set topology, and may be manufactured from the Stone space of suitable Booolean algebras, or by other means (cf. [2] for a recent and interesting example). The example we shall give is taken from Gillman and Jerison [4, Exercises 4N,6M], who unfortunately only consider the case $\mathfrak{R}=\mathfrak{N}_{0}$. We shall consider the case of general $\mathfrak{R}$, which introduces slight additional difficulties. We want to thank A. Hager for suggesting this and other examples of nonisolated $\mathfrak{N}-P$ points.

Let $Y$ be a set of cardinality $>\Re$. Let $x_{0} \in Y$. Topologize $Y$ as follows: A set $U \subseteq Y$ is open if $x_{0} \notin U$, or if $x_{0} \in U$ and $Y \sim U$ has cardinality $\leqq \Re$. This amounts to giving $Y \sim\left\{x_{0}\right\}$ the discrete topology, and letting neighborhoods of $x_{0}$ be complements of sets of cardinality $\leqq \Re$. Clearly a set $F$ is closed in $Y$ if $x_{0} \in F$, or if $x_{0} \notin F$ and the cardinality of $F$ is $\leqq \Re$. From this, we see immediately that $Y$ is normal. Clearly $x_{0}$ is a nonisolated $\mathfrak{N}-P$ point of $Y$. To see that $Y$ is not extremally disconnected, let $U$ be a subset of $Y$ such that $x_{0} \notin U$ and both $U$ and $Y \sim U$ have cardinality $>\Re$. Then $U$ is open, and the smallest closed set containing $U$ is $U \cup\left\{x_{0}\right\}$. But the closure of $U, U \cup\left\{x_{0}\right\}$, is not open, so $Y$ is not extremally discon- 
nected. By the Stone-Nakano theorem, $C_{b}(Y)$, the bounded continuous functions on $Y$, do not form a boundedly complete vector lattice.

On the other hand, it is easy to show directly that $C_{b}(Y)$ is a boundedly $\mathfrak{R}$-complete vector lattice. Clearly, $f: Y \rightarrow \boldsymbol{R}$ is continuous if and only if, given $\varepsilon>0$, there exists a set $F_{\varepsilon}$ of cardinality $\leqq \mathfrak{R}$ such that $\left|f(x)-f\left(x_{0}\right)\right|<\varepsilon$ for all $x \notin F_{\varepsilon}$. Suppose $\mathscr{F} \cong C_{b}(Y)$ has cardinality $\leqq \Re$. Let $f$ be the pointwise supremum of $\mathscr{F}$. Let $\varepsilon>0$. For each $h \in \mathscr{F}$, let $F_{h, \varepsilon}$ be a set of cardinality $\leqq \mathfrak{N}$ such that $\mid h(x)-$ $h\left(x_{0}\right) \mid<\varepsilon$ for $x \notin F_{h, \varepsilon}$. Let $F_{\varepsilon}=\bigcup\left\{F_{h, \varepsilon}: h \in \mathscr{F}\right\}$. The $F_{\varepsilon}$ has cardinality $\leqq \Re$, and if $x \notin F_{\varepsilon}$, then $\left|h(x)-h\left(x_{0}\right)\right| \leqq \varepsilon$ for all $h \in \mathscr{F}$, so $\left|f(x)-f\left(x_{0}\right)\right| \leqq \varepsilon$ as well. Thus $f$ is continuous, and so $C_{b}(Y)$ is boundedly $\mathfrak{R}$-complete.

Let $X$ be the Stone-Čech compactification of $Y$. Then $C(X)$ is isometrically isomorphic, and isomorphic as an ordered Banach space, to $C_{b}(Y)$. Thus $C(X)$ is not boundedly complete, so $X$ is not extremally disconnected. On the other hand, $C(X)$ is boundedly $\mathfrak{R}$ complete, so $X$ is totally disconnected and $\mathfrak{R}$-disconnected. Finally $Y$ is dense in $X$, so $x_{0}$, being an $\mathfrak{N}-P$ point of $Y$, is also an $\mathfrak{N}-P$ point of $X$. Clearly, $x_{0}$ is not an isolated point of $X$.

By taking finite disjoint unions of copies of $X$, we may construct compact Hausdorff spaces with at least $n$ nonisolated $\mathfrak{N}-P$ points, which are totally disconnected and $\mathfrak{N}$-disconnected, but not extremally disconnected. Incidentally, $C(X)$ is a good example of a Banach space which is $\mathfrak{R}$-injective, but not iujective in $\mathscr{B}_{1}$. So is $C_{0}(X)=$ $\left\{f \in C(X): f\left(x_{0}\right)=0\right\}$ ! Because $x_{0}$ is an $\mathfrak{R}-P$ point, $C_{0}(X)$ is a boundedly $\mathfrak{R}$-complete vector sublattice of $C(X)$, and so satisfies condition $a_{\mathfrak{n}}$. Hence, by Theorem 1 , it is $\Re$-injective. However, because $x_{0}$ is not isolated, $C_{0}(X)$ is not isometrically isomorphic to any $C(Z)$, and thus by the Goodner-Hasumi-Kelley-Nachbin theorem is not injetive in $\mathscr{B}_{1}$. We shall not go into greater detail, because this example will be subsumed under the promised general example, which we shall now give in the form of a theorem:

THEOREM 2. Let $X$ be a compact Hausdorff space which is totally disconnected and $\mathfrak{N}$-disconnected, and which contains $n$ nonisolated $\mathfrak{N}-P$ points, $x_{1}, \cdots, x_{n}$. Let $x_{0} \in X$, let $c_{1}, \cdots, c_{n} \in[-1,1)$, and let $B=\left\{f \in C(X): f\left(x_{i}\right)=c_{i} f\left(x_{0}\right), i=1, \cdots, n\right\}$. Assume $n \geqq 1$ and $x_{0}, x_{1}$, $x_{n}$ are all distinct. Then $B$ is $\mathfrak{R}$-injective. However if at least one $c_{i} \neq-1$, then $B$ is not isometrically isomorphic to $C(Z)$ for any compact Hausdorff space $Z$.

Proof. The key to the proof is the fact that for any $f \in C(X)$ and $c \in \boldsymbol{R},\{x: f(x)=c\}$ is a $G_{\delta}$. Thus $f$ is constant in a neighborhood 
of any $P$ point of $X$. Let $C$ be $\mathfrak{i}$-separable and let $A$ be a subspace of $C$. Let $T_{0}: A \rightarrow B$ be a bounded linear map. Let $V$ be the closure of $T_{0}(A)$, and let $\mathscr{K}$ be a dense subset of $V$ of cardinality $\leqq \Re$. For $k \in \mathscr{K}$, let $G_{i, k}$ be a neighborhood of the $\mathfrak{R}-P$ point $x_{i}$ in which $k$ is constant. Since there are at most $\mathfrak{R} G_{i, k}$ 's, the set $\bigcap\left\{G_{i, k}: k \in \mathscr{K}\right\}$ is also a neighborhood of $x_{\imath}$. For $i=1,2, \cdots, n$, let $G_{i}$ be a clopen neighborhood of $x_{i}$ contained in $\bigcap\left\{G_{i, k}: k \in \mathscr{L}\right\}$, and assume $x_{0} \notin G_{i}$ and $G_{i} \cap G_{j}=\varnothing$ for $i \neq j$. Since $\mathscr{K}$ is dense in $V$, not only is each $f \in \mathscr{K}$ constant on $G_{i}$, but so is each $f \in V$.

Let $Y=X \sim \mathrm{U}_{i=1}^{n} G_{i}$ and let $r: C(X) \rightarrow C(Y)$ be the restriction map. Clearly $Y$ is not only compact, but also open in $X$. Thus $Y$ is both totally disconnected and $\mathfrak{R}$-disconnected, and hence $C(Y)$ is boundedly $\mathfrak{R}$-complete and hence is $\mathfrak{R}$-injective. Consequently, $r T_{0}: A \rightarrow C(Y)$ has an extension $S: C \rightarrow C(Y)$ with the same norm. Define $T: C \rightarrow B$ as follows: $T(c)(y)=S(c)(y)$ on $Y$, and $T(c)(x)=$ $c_{i} S(c)\left(x_{0}\right)$ on $G_{i}$. Since each $G_{i}$ is clopen, $T(c) \in C(X)$. Clearly $T(C) \cong$ $B, T$ is linear, and $\|T(c)\|=\|S(c)\|$, so $\|T\|=\left\|T_{0}\right\|$. Finally, if $a \in A$, then $T(a)=T_{0}(a)$, since $T_{0}(a)$ is constant on each $G_{\imath}$. Thus $B$ is $\mathfrak{R}$-injective.

We still must show that $B$ is not isomorphic to any $C(Z)$ if some $c_{i} \neq-1$. Let $\mathscr{E}$ be the set of extreme points of the unit ball of $B^{*}$, the dual of $B$, and endow $\mathscr{E}$ with the weak * topology. Be renumbering the $x_{i}$ if necessary, we may assume $\left\{x_{i}: c_{i}=-1\right\}=$ either $\varnothing$ or $\left\{x_{p_{+1}}, \cdots, x_{n}\right\}$. In the former case, set $p=n$. Then $\mathscr{E}$ is homeomorphic to the union of two disjoint copies of $X \sim\left\{x_{1}, \cdots, x_{p}\right\}$ (corresponding to \pm point evaluations) with $x_{0}$ in one copy identified with $\left\{x_{p_{+1}}, \cdots, x_{n}\right\}$ in the other copy and vice-versa, if $p<n$. Since $x_{1}, \cdots, x_{p}$ are not isolated points of $X, \mathscr{E}$ is not compact. Therefore, $B$ cannot be isometrically isomorphic to a $C(Z)$ [3, p. 113].

The reader should note that if some of the $c_{\imath}$ 's are $<0$, then $B$ is not even a sublattice of $C(X)$. Actually, we can say even more. If $Z$ is a compact Hausdorff space and $\sigma: Z \rightarrow Z$ is a homeomorphism such that $\sigma^{2}$ is the identity map, then $C_{\sigma}(Z)=\{f \in C(Z): f \circ \sigma=-f\}$. If for some $c_{i_{0}}, c_{\imath_{0}} \neq 0$ and $-1<c_{i}<1$, then $B$ is not even isometrically isomorphic to any $C_{o}(Z)$ ! For the set $S$ of extreme points of the dual unit ball of $B$ which are in minimal facets of the dual unit ball is clearly all of $\mathscr{E}$, and point evaluation at $x_{i_{0}}$ clearly lies in the weak * closure of $\mathscr{E}$. But for all $b \in B$, we have $b\left(x_{i}\right)<\|b\|$. Thus $B$ cannot be isomorphic to any $C_{\sigma}(Z)$ by a theorem of Jerison's [3, p. 121].

Each $\Re$-Injective is Almost of Type $C(X)$.

Despite the example we have just given, an $\mathfrak{R}$-injective Banach space is not too far removed from a space of type $C(X)$. First, the 
spaces of type $C(X)$ share with the space of the example we have just given the property that their duals are isometrically isomorphic to a space of type $L^{1}(\mu)$. (A long list of spaces which are preduals of spaces of type $L^{1}(\mu)$ is given in [8, pp. 180-181]. (I would like to thank Y. Benjamini and the referee for bringing the class of preduals of spaces of type $L^{1}(\mu)$ to my attention.) A well known result of Lindenstrauss's states that each Banach space enjoying a finite dimensional extension property (which is much weaker than the extension property of $\mathfrak{R}$-injectivity) is the predual of an $L^{1}(\mu)$ space [9, Theorem 6.1]. Hence the $\mathfrak{R}$-injective Banach space and the spaces of type $C(X)$ all belong to the rather large family of preduals of spaces of type $L^{1}(\mu)$. But an $\mathfrak{R}$-injective space is more closely related to the spaces of type $C(X)$ than this. In fact, it turns out that if $B$ is $\Re$-injective, then $B$ is the direct limit of its $\mathfrak{N}$-separable subspaces of type $C(X)$.

We may prove this, and more, essentially by means of a slight modification of Kelley's original proof that a Banach space which is injective in the category $\mathscr{B}_{1}$ is of type $C(X)$ [7]. In what follows, if $E$ is a Banach space, then $E^{*}$ shall denote its dual, and $U_{E}$ shall denote the closed unit ball of $E$. If $K$ is a convex subset of $E$, then ext $K$ shall denote the set of extreme points of $K$. If $Y \subseteq E$, then Cl $Y$ shall denote the closure of $Y$. The topology with respect to which the closure is taken will be specified whenever it is not clear from context. Finally, if $Y$ is a compact Hausdorff space and $y \in Y$, then $e_{y} \in C(Y)^{*}$ shall denote evaluation at the point $y$.

Lemma 1. Let $M$ and $N$ be Banach spaces, and let $S: M \rightarrow N$ be a linear map of norm $\leqq 1$. Let $p$ be an extreme point of $U_{N}$, and let $L=S^{-1}(p) \cap U_{M}$. Then either $L=\varnothing$ or $L$ is a support of $U_{M}$.

Lemma 1 is a standard fact (cf. [7]).

Theorem 3. Let $B$ be an $\Re$-injective Banach space. Let $A$ be an $\mathfrak{N}$-separable subspace of $B$, and let $i: A \subset B$ be the inclusion map. Let $W$ be a weak * relatively open subset of $\mathrm{Cl}$ ext $U_{A^{*}}$, such that $W \cap(-W)=\varnothing$ and $\mathrm{Cl}(W \cup(-W))=\mathrm{Cl} \operatorname{ext} U_{A^{*}}$. Let $Y=\mathrm{Cl} W$. Here, the closures are taken with respect to the weak * topology. Endow $Y$ with the weak * topology, and let $j: A \rightarrow C(Y)$ be the natural isometric injection. Then there exists an isometric injection $p: C(Y) \rightarrow B$ such that $p \circ j=i$.

Before proving Theorem 3, three comments are in order. First, as Kelley observed, it is easy to produce such sets $W$ : Simply apply Zorn's lemma to produce a set $W$ which is maximal with respect to 
the two properties $W \cap(-W)=\varnothing$, and $W$ is weak $*$ open in $\mathrm{Cl} \operatorname{ext} U_{A^{*}}$ [7]. Second, from the Krein-Milman theorem, we know that if $a \in A$, then $\sup \left\{y(\alpha): y \in U_{A^{*}}\right\}$ is actually attained at some $y \in \mathrm{C} 1$ ext $U_{A^{*}}$. Since $W \cap(-W)$ is dense in C1 ext $U_{A^{*}}$, it follows that $j$ is an isometry. Finally, $Y$ is clearly compact by the Alaoglu theorem.

Proof. First, we must show that $C(Y)$ is $\Re$-separable. Observe that $j(A)$ is $\mathfrak{N}$-separable, so the subalgebra in $\mathscr{A}$ in $C(Y)$ genarated by $j(A)$ and the function $\equiv 1$ is also $\Re$-separable. But $\mathscr{A}$ separates points of $Y$ because $A$ does. By the Stone-Weierstrass theorem, $\mathscr{A}$ is dense in $C(Y)$, so $C(Y)$ is also $\mathfrak{R}$-separable. From the $\mathfrak{R}$-injectivity of $B$, we conclude that there exists a linear map $p: C(Y) \rightarrow B$ of norm 1 such that $p \circ j=i$.

We will show that $p$ is $1-1$ by showing that its adjoint $p^{*}: B^{*} \rightarrow C(Y)^{*}$ is onto. We assert that it suffices to show that $\left\{e_{y}: y \in W \cap \operatorname{ext} U_{A^{*}}\right\} \subseteq p^{*}\left(U_{B^{*}}\right)$. To see why, suppose this inclusion holds. We know $p^{*}$ is weak $*$ continuous and $U_{B^{*}}$ is weak $*$ compact, so $p^{*}\left(U_{B^{*}}\right)$ is weak * compact and hence weak * closed in $C(Y)^{*}$. Thus $\mathrm{Cl}\left\{e_{y}: y \in W \cap \operatorname{ext} U_{A^{*}}\right\} \cong p^{*}\left(U_{B^{*}}\right)$. But the map $y \rightarrow e_{y}, y \in Y$, is a homeomorphism from $Y$ onto the set $\left\{e_{y}: y \in Y\right\}$ endowed with the weak * topology. Furthermore, because $W$ is an open subset of $\mathrm{Clext} U_{A^{*}}$, we know that $\mathrm{Cl}\left(W \cap \operatorname{ext} U_{A^{*}}\right)=\mathrm{Cl} W=Y$. Thus $\left\{e_{y}: y \in Y\right\} \cong p^{*}\left(U_{B^{*}}\right)$. From this, we conclude that ext $U_{C^{\prime} Y^{*}}=$ $\left\{ \pm e_{y}: y \in Y\right\}$, as well as the closed convex hull of ext $U_{C(Y)^{*}}$, are contained in $p^{*}\left(U_{B^{*}}\right)$. By the Krein-Milman theorem, $U_{C\left(Y^{*}\right)} \subseteq p^{*}\left(U_{B^{*}}\right)$. Thus $p^{*}$ is onto.

In fact, from this last inclusion, we may conclude that $p$ is not only $1-1$, but is actually an isometry. Suppose $p$ were not an isometry. We know that $\|p\|=1$, so there exists $f \in C(Y)$ such that $\|f\|_{1}=1$ and $\|p(f)\|<1$. Let $\mu \in C(Y)^{*}$ be a linear functional of norm 1 such that $\mu(f)=1$. If $\lambda \in U_{B^{*}}$, then $\left|p^{*}(\lambda)(f)\right|=|\lambda(p(f))| \leqq$ $\|p(f)\|<1$, so $\mu \notin p^{*}\left(U_{B^{*}}\right)$. This is a contradiction.

We thus need only show that $\left\{e_{y}: y \in W \cap\right.$ ext $\left.U_{A^{*}}\right\} \subseteq p^{*}\left(U_{B^{*}}\right)$ in order to complete the proof of Lemma 2. We will do this by chasing the following commutative diagram of adjoint maps:

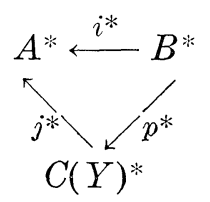

Note that all of the maps involved have norm $\leqq 1$. Let $y \in Y$. Clearly $j^{*}\left(e_{y}\right)=y$ for each $y \in Y$. We assert that if $y \in W \cap \operatorname{ext} U_{A^{*}}$, 
then $j^{*-1}(y) \cap U_{C(Y)^{*}}=\left\{e_{y}\right\}$. Let $L=j^{*-1}(y) \cap U_{C(Y)^{*}}$. By Lemma 1, $L$ is a support of $U_{C(Y) *} \quad L$ is closed in $U_{C(Y) *}$ and hence is compact. By the Krein-Milman theorem, $L$ has extreme points. Because $L$ is a support of $U_{C(Y) *}$, each extreme point $w$ of $L$ is also an extreme point of $U_{C(Y)^{*}}$, and hence is of the form $\pm e_{z}$ for some $z \in Y$. If $w=-e_{z}$, then for each $a \in A$ we have $y(a)=j^{*}\left(-e_{z}\right)(a)=-e_{z}(j(a))=$ $-z(a)$, so $y=-z$. Hence $y \in W \cap(-\mathrm{C} 1 W)$. But $W \cap(-\mathrm{C} 1 W)=\varnothing$, since $W \cap(-W)=\varnothing$ and $W$ is open. Thus $w$ is not $=-e_{z}$ for any $z \in Y$. If $w=e_{z}$ for some $z \in Y$, it is immediate that $z=y$. Hence the only extreme point of $L$ is $e_{y}$. By the Krein-Milman theorem, $L=\left\{e_{y}\right\}$.

Now let $y \in W \cap \operatorname{ext} U_{A^{*}}$, and observe that $i^{*-1}(y) \cap U_{B^{*}} \neq \varnothing$ by the Hahn-Banach theorem. Let $z \in i^{*-1}(y) \cap U_{B^{*}}$. By the commutativity of diagram $2, j^{*} p^{*}(z)=i^{*}(z)=y$, so $p^{*}(z) \in j^{*-1}(y) \cap U_{B^{*}}$, and hence $p^{*}(z)=e_{y}$. Consequently, $\left\{e_{y}: y \in W \cap \operatorname{ext} U_{A^{*}}\right\} \subseteq p^{*}\left(U_{B^{*}}\right)$, which completes the proof of Theorem 3 .

CoRollary 1. Suppose $B$ is not only $\mathfrak{R}$-injective, but also $\mathfrak{R}$ separable. Then $B$ is isometrically isomorphic to $C(X)$, for some extremally disconnected compact Hausdorff space $X$. Hence $B$ is injective in the category $\mathscr{B}_{1}$.

Proof. In the statement of Theorem 3, choose $A=B$ and $X=Y$. Then $B$ is isometrically isomorphic to $C(X)$. By Theorem 1, Corollary $1, C(X)$ satisfies condition $a_{\mathfrak{r}}$. But $C(X)$ is $\mathfrak{N}$-separable, so $C(X)$ satisfies condition $a_{\Re^{\prime}}$ for every infinite cardinal $\mathfrak{N}^{\prime}$, and thus $C(X)$ is a boundedly complete vector lattice. The remainder of the corollary now follows from Theorem 1, Corollary 2, and the Stone-Nakano theorem.

Corollary 1 may be rephrased to assert that if $B$ is injective in the full subcategory of $\mathscr{B}_{1}$ whose objects are all $\mathfrak{N}$-separable spaces, then $B$ is actually injective in $\mathscr{B}_{1}$. As we mentioned in the introduction, Corollary 1 is interesting because the situation is dramatically different in the category $\mathscr{B}$. It would be interesting to know if there are any nontrivial full subcategories of $\mathscr{B}_{1}$ in which new injectives can arise. As we shall see in Corollary 5, the full subcategory $\mathscr{C}_{1}$ of $\mathscr{B}_{1}$, whose objects are all weakly compactly generated spaces, is not such a subcategory.

CoRollary 2. $B$ is injective in the category $\mathscr{B}_{1}$ if and only if $B$ is isometrically isomorphic to $C(X)$, for some extremally disconnected compact Hausdorff space $X$. 
Proof. One way is Theorem 1, Corollary 2. Conversely, suppose $B$ is injective in $\mathscr{B}_{1}$. $B$ is $\mathfrak{R}$-separable for some $\mathfrak{N}$, and also $\mathfrak{R}$ injective. Apply Corollary 1.

Corollary 2 is the full Goodner-Kelley-Nachbin characterization of injectives in $\mathscr{B}_{1}$ [5], [7], [11]. Of course, Corollary 2 is a bit of a cheat, because the proof of Theorem 3 is essentially Kelley's proof (slightly modified) that each injective in $\mathscr{B}_{1}$ is of type $C(X)$. However, the following corollary is somewhat more interesting because it may provide the first step toward a complete characterization of $\mathfrak{R}$ injectives.

Corollary 3. Let $B$ be an $\mathfrak{R}$-injective Banach space. Then $B$ is the direct limit of its $\mathfrak{N}$-separable subspaces of type $C(Y)$.

Proof. It suffices to prove that $B$ is the union of such subspaces, and that if $A$ and $C$ are two such $\Re$-separable subspaces, then there is an $\Re$-separable subspace $D$ of type $C(Y)$ such that $A \cup C \leqq D$. Both assertion follow immediately from Theorem 3.

I am indebted to Y. Benjamini for pointing out that Corollary 1 is actually strong enough to imply the following important result:

Corollary 4. There are no $\mathfrak{R}$-injective, infinite dimensional, weakly compactly generated spaces.

Proof. Suppose $B$ is an $\mathfrak{M}$-injective space which is also infinite dimensional and weakly compactly generated. Let $E$ be an infinite dimensional separable subspace of $B$. By a fundamental result of Lindenstrauss's, there exists a separable subspace $D \supseteqq E$ and a projection $P: B \rightarrow D$ of norm 1 [10, pp. 170-171]. The existence of the projection $P$ guarantees that $D$ is $\mathfrak{R}$-injective. By Corollary $1, D$ is isometrically isomorphic to $C(X)$, where $X$ is extremally disconnected and compact Hausdorff. Since $C(X)$ is separable, $X$ is metrizable. Thus $X$ has finite cardinality, so $D=C(X)$ is finite dimensional, which is impossible.

COROLlary 5. The only injectives in $\mathscr{C}_{1}$ are already injective in the larger category $\mathscr{B}_{1}$, and are in fact finite dimensional.

Proof. Let $B$ be an injective in $\mathscr{C}_{1}$. Since $\mathscr{C}_{1}$ contains every separable space, $B$ is $\Re_{0}$-injective. Apply Corollary 4 .

In conclusion, we would like to raise a question whose resolution probably awaits a complete characterization of $\mathrm{N}$-injectives. Cohen showed that every Banach space has an injective envelope in the 
catogory $\mathscr{B}_{1}[1]$. It would be interesting to know whether or not every Banach space also has an $\mathfrak{R}$-injective envelope.

\section{REFERENCES}

1. H. B. Cohen, Injective envelopes of Banach spaces, Bull. Amer. Math. Soc., 70 (1964), 723-726.

2. W. W. Comfort and Anthony W. Hager, Uniform Continuity in Topological Groups, to appear.

3. M. M. Day, Normed Linear Spaces, 2nd ed., Ergebnisse der Mathematik und Ihrer Grenzgebiete, Springer, Berlin, 1972.

4. L. Gillman and M. Jerison, Rings of Continuous Functions, Van Nostrand, New York, 1960.

5. D. B. Goodner, Projections in normed linear spaces, Trans. Amer. Math. Soc., 69 (1950), 89-108.

6. M. Hasumi, The extension property of complex Banach spaces, Tôhoku Math, J., 10 (1958), 135-142.

7. J. L. Kelley, Banach spaces with the extension property, Trans. Amer. Math. Soc., 72 (1952), 323-326.

8. A. J. Lazar and J. Lindenstrauss, Banach spaces whose duals are $L_{1}$ spaces and their representing matrices, Acta Math., 126 (1971), 165-193.

9. J. Lindenstrauss, Extension of compact operators, Mem. Amer. Math. Soc., 48 (1964).

10. Some aspects of the theory of Banach spaces, Adv. in Math., 5 (1970), 159-180.

11. L. Nachbin, A theorem of the Hahn-Banach type for linear transformations, Trans. Amer. Math. Soc., 68 (1950), 28-46

12. M. H. Stone, Boundedness properties in function-lattices, Canad. J. Math., 1 (1949), 176-186

13. W. A. Veech, Short proof of Sobczyk's theorem, Proc. Amer. Math. Soc., 28 (1971), 627-628.

Received April 14, 1975. I wish to thank Anthony Hager for several extremely helpful conversations about $P$-points and the Stone-Nakano theorem, and Y. Benjamini for several extremely helpful conversations about preduals of $L^{1}(\mu)$ spaces and weakly compactly generated Banach spaces.

Central Connecticut State College 


\section{PACIFIC JOURNAL OF MATHEMATICS}

EDITORS

RICHARD ARENS (Managing Editor)

University of California

Los Angeles, California 90024

R. A. BEAUMONT

University of Washington

Seattle, Washington 98105
J. DugundJI

Department of Mathematics

University of Southern California

Los Angeles, California 90007

D. Gilbarg and J. Milgram

Stanford University

Stanford, California 94305

\section{ASSOCIATE EDITORS}

E. F. BECKENBACH

B. H. NeumanN

F. WOLF

K. YosHIDA

\section{SUPPORTING INSTITUTIONS}

UNIVERSITY OF BRITISH COLUMBIA

UNIVERSITY OF SOUTHERN CALIFORNIA

CALIFORNIA INSTITUTE OF TECHNOLOGY

UNIVERSITY OF CALIFORNIA

STANFORD UNIVERSITY

UNIVERSITY OF TOKYO

MONTANA STATE UNIVERSITY

UNIVERSITY OF UTAH

UNIVERSITY OF NEVADA

WASHINGTON STATE UNIVERSITY

NEW MEXICO STATE UNIVERSITY

UNIVERSITY OF WASHINGTON

OREGON STATE UNIVERSITY

UNIVERSITY OF OREGON

OSAKA UNIVERSITY

AMERICAN MATHEMATICAL SOCIETY
NAVAL WEAPONS CENTER

Printed in Japan by International Academic Printing Co., Ltd., Tokyo, Japan 


\section{Pacific Journal of Mathematics}

\section{Vol. 63, No. $1 \quad$ March, 1976}

Ralph Artino, Gevrey classes and hypoelliptic boundary value problems ....... 1

B. Aupetit, Caractérisation spectrale des algèbres de Banach commutatives .... 23

Leon Bernstein, Fundamental units and cycles in the period of real quadratic

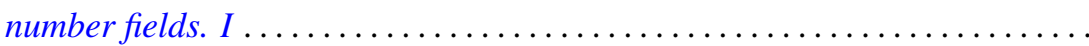

Leon Bernstein, Fundamental units and cycles in the period of real quadratic number fields. II.................................... 63

Robert F. Brown, Fixed points of automorphisms of compact Lie groups ........

Thomas Ashland Chapman, Concordances of noncompact Hilbert cube

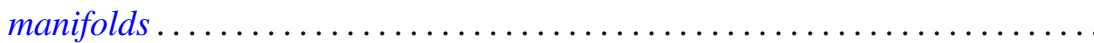

William C. Connett, V and Alan Schwartz, Weak type multipliers for Hankel

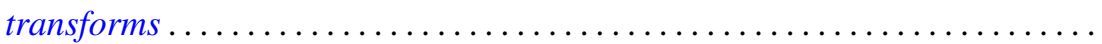

John Wayne Davenport, Multipliers on a Banach algebra with a bounded approximate identity .....................................

Gustave Adam Efroymson, Substitution in Nash functions ................ 137

John Sollion Hsia, Representations by spinor genera ..................

William George Kitto and Daniel Eliot Wulbert, Korovkin approximations in

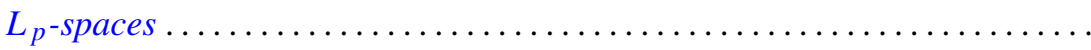

Eric P. Kronstadt, Interpolating sequences for functions satisfying a Lipschitz. condition ...........................................

Gary Douglas Jones and Samuel Murray Rankin, III, Oscillation properties of certain self-adjoint differential equations of the fourth order...

Takaŝi Kusano and Hiroshi Onose, Nonoscillation theorems for differential

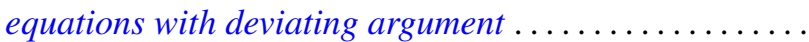

David C. Lantz, Preservation of local properties and chain conditions in commutative group rings. ...

Charles W. Neville, Banach spaces with a restricted Hahn-Banach extension property....

Norman Oler, Spaces of discrete subsets of a locally compact group ...

Robert Olin, Functional relationships between a subnormal operator and its minimal normal extension.

Thomas Thornton Read, Bounds and quantitative comparison theorems for nonoscillatory second order differential equations ...... .

Robert Horace Redfield, Archimedean and basic elements in completely distributive lattice-ordered groups...

Jeffery William Sanders, Weighted Sidon sets

Aaron R. Todd, Continuous linear images of pseudo-complete linear topological spaces.

J. Jerry Uhl, Jr., Norm attaining operators on $L^{1}[0,1]$ and the Radon-Nikodým property.

William Jennings Wickless, Abelian groups in which every endomorphism is a left multiplication. 